\title{
Viewpoint
}

\section{Ups and Downs in the Search for Dark Matter}

\author{
Rafael F. Lang \\ Department of Physics, Purdue University, West Lafayette, IN 4790\%, USA
}

Published December 16, 2013

Two leading dark-matter-detection experiments find conflicting results in the search for dark matter.

Subject Areas: Particles and Fields, Astrophysics, Cosmology

\author{
A Viewpoint on: \\ Silicon Detector Dark Matter Results from the Final Exposure of CDMS II \\ R. Agnese emet al./em (CDMS Collaboration) \\ Physical Review Letters 111, 2513012013 - Published December 16, 2013
}

First Results from the LUX Dark Matter Experiment at the Sanford Underground Research Facility D. S. Akerib emet al./em (LUX Collaboration)

Physical Review Letters 112, 0913032014 - Published March 4, 2014

We know dark matter is out there. Astrophysical observations of gravitational effects provide evidence of its existence at all times, from the time of nucleosynthesis (a few minutes after the big bang) to the moment in which the cosmic microwave background radiation was released (300,000 years after the big bang), all the way to the present Universe. Dark matter pervades all length scales, from the Universe as a whole to individual galaxies, including our Milky Way, and even to some smaller structures. The question is not, does dark matter exist? The question is, what is dark matter made of?

In separate reports, one appearing in Physical Review Letters [1, the other on the arXiv [2], two collaborations - the Cryogenic Dark Matter Search (CDMS) experiment at Fermilab in Illinois and the Large Underground Xenon (LUX) experiment in South Dakota - present the results from their searches for dark matter candidate particles called weakly interacting massive particles (WIMPs). Both collaborations attempt to directly detect WIMPs as they scatter off the nuclei of a detector. CDMS finds a signal that could potentially be assigned to WIMPs. But LUX, with nominally better sensitivity, sees no evidence of such dark matter particles.

WIMPs were first proposed in the 1980 s, with masses expected above $\sim 100$ giga-electron-volts (about a hundred times heavier than a proton) and interactions mediated through the weak force, i.e., through the exchange of $Z$ or $W$ bosons. One candidate for the WIMP was the heavy neutrino, which theorists expected to scatter from nucleons with a cross section in the range of $10^{-38} \mathrm{~cm}^{2}$ - a value that could have allowed their observation. But searches for such particles in the 1990s came up empty handed. Researchers have thus proposed alternative options: The interaction could be mediated by the Higgs boson or by some yet unknown mediator,

DOI: $10.1103 /$ Physics.6.136

URL: http://link.aps.org/doi/10.1103/Physics.6.136 yielding expected scattering cross sections about 10 orders of magnitude below those expected from heavy neutrinos. Another type of WIMP, with a mass expected to be much lighter [only a few giga-electron-volts $\left(\mathrm{GeV} / \mathrm{c}^{2}\right)$ ], was invoked to explain the apparent particle-antiparticle asymmetry of the Universe [3]. Probing these parameter regions is the challenge posed to experiments aiming at the direct detection of dark matter (see Fig. 1).

The direct-detection experiments confront two main challenges. One is the low energy of the interaction: The energy released when a WIMP scatters from a detector nucleus depends on the WIMP mass and the speed with which it hits the detector. Given the nonrelativistic velocities characteristic of our galaxy (e.g., we go around the Milky Way at about $230 \mathrm{~km} / \mathrm{s}$ ), the deposited energy can be expected to be, at most, a few kilo-electronvolts, truly low for a particle detector. A second difficulty is that the expected signal spectrum is a smooth, steeply falling function of increasing energy. This concentrates the expected signal exclusively near the lower energy threshold of detectors.

The CDMS collaboration has spearheaded the search for dark matter over much of the last decade. CDMS detectors are stacks of hockey-puck-sized ultrapure germanium (Ge) or silicon ( $\mathrm{Si}$ ) crystals operated at temperatures of only 40 millikelvin. If a particle scatters in one of these crystals, it induces a nuclear recoil that can be measured. The interaction energy is determined essentially by measuring the resulting temperature rise in the detector. In addition, the detectors are operated as ionization detectors so that each interaction also yields a second signal. For a given energy, this ionization signal is different depending on whether the interaction is due to an electronic recoil (from background beta or gamma radioactivity) or a nuclear recoil (from neutrons or WIMPs). 


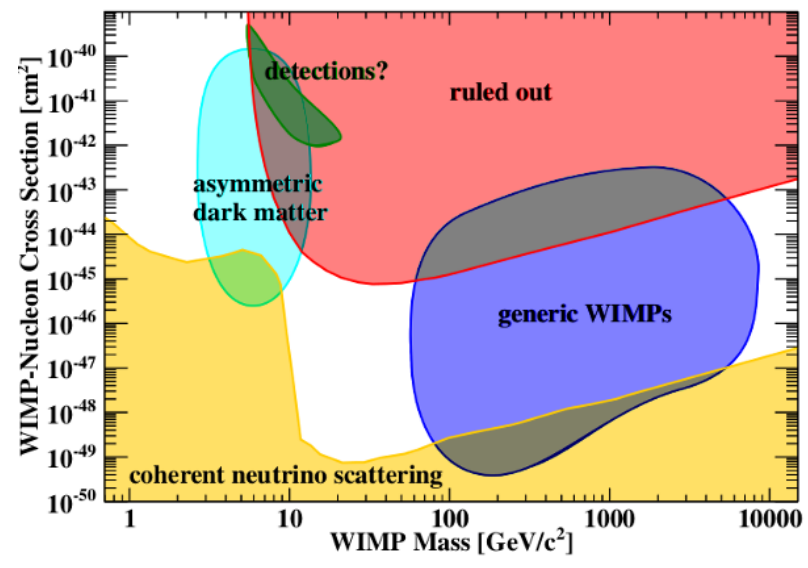

FIG. 1: Simplified plot showing the predicted WIMP-nucleon scattering cross section as a function of WIMP mass. Asymmetric dark matter models (light blue area) predict WIMPs with masses of a few $\mathrm{GeV} / \mathrm{c}^{2}$; generic WIMP models (dark blue area) predict larger masses of hundreds of $\mathrm{GeV} / \mathrm{c}^{2}$ or more. Parts of these parameter regions have been probed and excluded by current experiments (red area). The signal excess of the CDMS experiment points towards relatively light WIMPs (green area). However, the absence of a signal in the LUX experiment is in tension with this result. Most of the expected parameter space could be probed in the near future, until a background from coherent neutrino-nucleus scattering (yellow area) becomes relevant, interfering with possible dark matter signals. (APS/Rafael Lang)

This allows the signal of a nuclear recoil to be efficiently discriminated from the background of electronic recoils. In their paper, CDMS presents the result from a search over more than a year using eight of their Si detectors, with a total exposure of $140 \mathrm{~kg}$.days. (The experimental exposure of these experiments is given in units of target mass times measurement live time, $\mathrm{kg}$.days.) With this exposure, CDMS would have expected 0.6 events in a predefined signal region from known backgrounds. They observed three.

This is intriguing. It is the first report of an excess over background from a dark matter search referred to as "zero-background" experiment - an experiment that, in a given signal region, should yield less than 1 event due to known background sources. This type of experiment offers the most efficient search strategy, as it is not limited by background counting statistics but only by the exposure time itself. Given the low recoil energy of the observed excess and the lightness of the CDMS Si target, a dark matter interpretation points towards relatively light WIMPs with masses below $10 \mathrm{GeV} / \mathrm{c}^{2}$ (as expected, e.g., in so-called asymmetric dark matter models [3]). Not surprisingly then, this CDMS result has been met with immense interest and has spurred many speculations about whether this excess could be a genuine dark matter signal.

The result of the LUX experiment dampens this excitement. LUX uses a cryostat filled with $370 \mathrm{~kg}$ of xenon

DOI: 10.1103/Physics.6.136

URL: http://link.aps.org/doi/10.1103/Physics.6.136 from which they currently use the innermost $118 \mathrm{~kg}$ to search for dark matter. Liquid xenon is an excellent scintillator: Any particle interaction results in a prompt flash of light that is observed by an array of photomultiplier tubes. Application of an electric field allows the detection of the ionization generated by the interaction, too. Just like in CDMS, analyzing these two signals allows experimentalists to discriminate between electronic and nuclear recoils. In addition, this detector also measures the position of the interaction. With this technology, more massive detectors can be built, enhancing the probability of detecting a WIMP. In addition, the LUX experiment is sensitive to the position of the interaction in the detector. Hence, events happening near the surfaces of the target can be ignored based on their position. This reduces the impact of detector surface areas, which exhibit larger radiation-induced backgrounds and are hard to understand. During a first run over four months, the LUX collaboration has searched for dark matter with an exposure of about $10,000 \mathrm{~kg} \cdot$ days. They observed no signal excess in their signal parameter range, where hundreds of events would have been expected if the CDMS excess had been due to WIMPs. In particle physics, "blind" analysis strategies aim to remove unintended influence on a measurement from prior expectations of the experimentalists, and although the LUX analysis was, in contrast to CDMS, not blind, this would seem to rule out the possibility that CDMS has seen dark matter. Or does it?

There is some wiggle room for reconciling both experiments. For example, both have to make assumptions about how dark matter is distributed in the Milky Way or how precisely WIMPs interact with nuclei. Great care has to be taken to accurately measure the efficiencies of these experiments in revealing WIMP-induced events, using an array of methods ranging from accurate simulation of the detectors to calibrations with dedicated neutron sources. Studies that explore how these assumptions affect the interpretation of the two experiments are already being published on a weekly basis.

Regardless of the outcome of such studies, what we are witnessing is an example of how the identification of dark matter will come about. We cannot expect a claim, beyond any statistical or systematic doubt, from a single experiment, but rather a gradual process. At some point there will be a barely significant excess over known backgrounds that, despite careful experimental efforts, fails to go away. At that point, other experiments with complementary technologies have to be available in order to confirm or rule out any dark matter claim. Only with multiple experiments, different detection technologies, and various target materials at hand, can we hope to undoubtedly claim a dark matter signal and unravel the properties of dark matter [4].

It is remarkable that our experiments today are of such minute sensitivity that any signal excess could very reasonably be due to dark matter [5]. We are probing the most relevant areas of parameter space where WIMPs are expected, and any day, we could start seeing the tip of 
the dark matter iceberg. For well over a decade, experiments aimed at direct detection of WIMPs have easily beaten Moore's law by roughly doubling their sensitivity every year. We can only hope that the relatively minor resources that are required for solving one of the most important outstanding problems in physics will continue to be available for supporting these efforts.

A number of other collaborations are racing to present results from their experiments. And of course, both the CDMS and LUX collaborations are moving ahead to acquire better data. CDMS now runs CDMSLite, a dedicated search to improve their sensitivity in particular to light WIMPs. In addition, they deploy advanced detectors with far superior interleaved electrodes to reduce the unwanted effects of surfaces [6]. LUX is also improving various technical aspects of their detector and is running to accumulate more exposure in the race to find dark matter.

We will soon reach the sensitivities in which our experiments will be sensitive to a never-observed process (see Fig. 1): coherent neutrino-nucleus scattering, induced by neutrinos coming from the sun, the atmosphere, or supernovae [7]. An intriguing signal in its own right, this process will pose an unavoidable background contribution that might hamper dark matter detection. If we have failed to identify dark matter by then, it will be time to go back to the drawing board. But at least we will have done the best we could to tease out one of nature's biggest secrets.

Correction (5 March 2014): Reference 2 was updated to show that the LUX Collaboration's paper published in Physical Review Letters.

\section{References}

[1] R. Agnese et al. (CDMS Collaboration), "Silicon Detector Dark Matter Results from the Final Exposure of CDMS II," Phys. Rev. Lett. 111, 251301 (2013).

[2] D.S. Akerib et al. ((LUX Collaboration)), "First Results from the LUX Dark Matter Experiment at the Sanford Underground Research Facility," Phys. Rev. Lett. 112, 091303 (2014).

[3] D. E. Kaplan, M. A. Luty, and K. M. Zurek, Phys. Rev. D 79, 115016 (2009).

[4] A. H. G. Peter, V. Gluscevic, A. M. Green, B. J. Kavanagh, and S. K. Lee, "WIMP Physics with Ensembles of Direct-Detection Experiments," arXiv:1310.7039 (2013).

[5] P. Cushman et al., "Snowmass CF1 Summary: WIMP Dark Matter Direct Detection," arXiv:1310.8327.

[6] R. Agnese et al., "Demonstration of Surface Electron Rejection with Interleaved Germanium Detectors for Dark Matter Searches," Appl. Phys. Lett. 103, 164105 (2013).

[7] J. Billard, E. Figueroa-Feliciano, and L. Strigari, "Implication of Neutrino Backgrounds on the Reach of Next Generation Dark Matter Direct Detection Experiments," arXiv:1307.5458 (2013).

\section{About the Author}

\section{Rafael F. Lang}

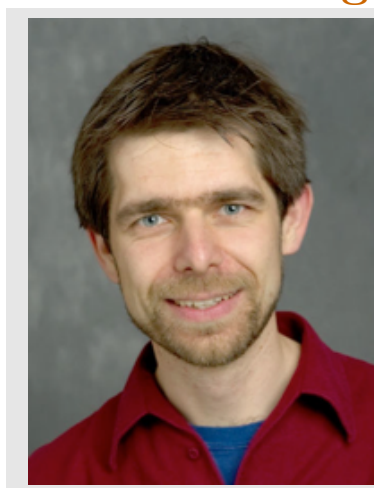

Rafael Lang is Assistant Professor at Purdue University, Indiana. He is working on the XENON dark matter search project, using a cryostat filled with liquid xenon to find dark matter in the Gran Sasso underground laboratory, Italy. For his Ph.D., he was working at the Max-Planck-Institut für Physik in München on the CRESST experiment, using scintillating crystals at millikelvin temperatures to search for dark matter. From there, his postdoc took him to Columbia University, New York, also on the XENON project, before joining Purdue. For contact information, visit: http://www.physics.purdue.edu/people/faculty/lang. shtml. 